\section{P134 COMPARISON OF TWO PROGNOSTIC TOOLS FOR IDENTIFYING HIGH RISK PATIENTS WITH IDIOPATHIC PULMONARY FIBROSIS}

doi:10.1136/thoraxjnl-2012-202678.417

MEM Wilkie, JD Chalmers, RP Smith, S Schembri. NHS Tayside, Dundee, United Kingdom

Introduction Idiopathic pulmonary fibrosis (IPF) is a progressive disease with median survival of $2-3$ years. Clinical course and survival is variable. Whilst no universally accepted tool is accessible to identify those with poorer prognosis, du Bois et $\mathrm{al}^{(1)}$ and Ley et $\mathrm{al}^{(2)}$ propose individual 4 point scoring systems to calculate mortality. Our aim was to compare both tools and assess for superiority.

Methods Data was collected on 101 patients retrospectively, 13 were excluded. The du Bois score included age, respiratory hospitalisation, baseline forced vital capacity (FVC) and 6 month change in FVC. ${ }^{(1)}$ The GAP index used gender, age, FVC and DLCO. (2) Predictive values were assessed using area under the receiver operator characteristic curve (AUC). We used GAP index $>1$ and du Bois score $>22$ to indicate high risk for calculation of performance characteristics. Patients were followed up for 3 years or until death.

Results Median age was 69 years (interquartile range 63-76) $57.5 \%$ were male. 1 year mortality was $10.2 \%$, compared with $18.2 \%$ at year 3 . For prediction of 1 year mortality, the AUC was $0.78(0.69-0.87)$ for the du Bois score and $0.73(0.63-0.84)$ for the GAP index. For 3 year mortality, the AUC was $0.73(0.65-0.80)$ for both systems. There was no significant difference between the scores for either outcome $(p=0.5$ and $p=0.9$ respectively). The receiver operator characteristic curves for 1 year mortality are shown: figure 1.

For 1 year mortality, the GAP index had a sensitivity of $87 \%$, specificity $55 \%$ compared to $66.7 \%$ and $71.1 \%$ for the du Bois score. For 3 year mortality, the GAP index had a sensitivity of $86.7 \%$, specificity $58.9 \%$ compared to $62.5 \%$ and $73.7 \%$ for the du Bois score.

Conclusions This study shows that both systems are of equal value at identifying patients with IPF at high risk of mortality. References

1. du Bois RM et al. Ascertainment of individual risk of mortality for patients with idiopathic pulmonary fibrosis. Am J Respir Crit Care Med 2011; 184:459-466.

2. Ley B et al. A multidimensional index and staging system for idiopathic pulmonary fibrosis. Ann Intern Med. 2012; 156:684-691.

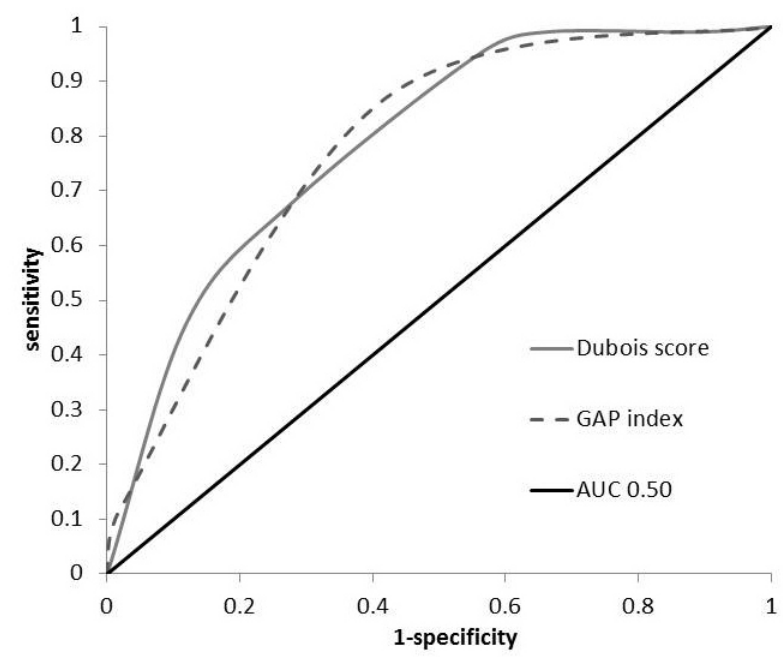

Abstract P134 Figure 1

\section{P135 QUALITY OF LIFE AND ADVERSE EFFECTS IN PATIENTS TAKING PIRFENIDONE FOR IDIOPATHIC PULMONARY FIBROSIS}

doi:10.1136/thoraxjnl-2012-202678.418

J Capps, B Amies, A Duck, R Frank, C Leonard, J Holme. University Hospital South Manchester, Manchester, UK

Introduction and Objectives Pirfenidone is currently the only disease-modifying therapy for idiopathic pulmonary fibrosis. However the CAPACITY ${ }^{1}$ study demonstrated frequent mild adverse effects. No data is available on the overall effect of pirfenidone on quality of life. Our aim was to describe patients' experience of pirfenidone, taking these factors in to account.

Methods This was a single-centre observational study of patients participating in the pirfenidone named patient programme. Participants completed the Self-Report Chronic Respiratory Disease Questionnaire (SR-CRQ), a quality of life assessment with four domains, at three monthly intervals. Adverse effects were also recorded. Patients were followed up until death or cessation of pirfenidone.

Results 26 patients were included, with 9 patients reaching 3 months, 6 reaching 6 months, and 5 reaching 9 months during the study period. Mean age was $67 \pm 7$ years, and $77 \%$ were male. There was a downward trend in all domains of the SR-CRO, as illustrated in figure 1 . However there were no significant differences in scores from baseline compared with individual time points. No relationship was found between SR-CRQ scores and adverse effects.

Adverse effects were experienced by 14 patients (54\%). These included gastrointestinal symptoms $(n=12)$, photosensitivity $(n=2)$ and fatigue $(n=3) .5$ patients stopped pirfenidone as a result of adverse effects. The mean weight in kilogrames of those patients experiencing adverse effects was lower than those who did not $(72.5 \pm 12.7$ vs $86.1 \pm 13.2, p=0.014)$. Age in years was higher in those with adverse effects $(70 \pm 6$ vs $64 \pm 8, p=0.035)$ Patients with more severe disease (FVC $<50 \%$, DLCO $<35 \%$ ) were also significantly more likely to have adverse effects $(p=0.005)$.

Conclusion The data from this study is limited due to small numbers, however we have not observed a significant change in quality of life from baseline in those treated with pirfenidone. Adverse effects were greater in older, lighter patients with more severe disease.

1. Noble P, Albera C, Bradford W et al, for the CAPACITY Study Group. Pirfenidone in patients with idiopathic pulmonary fibrosis (CAPACITY): two randomised trials. Lancet 2011; 377: 1760-69.

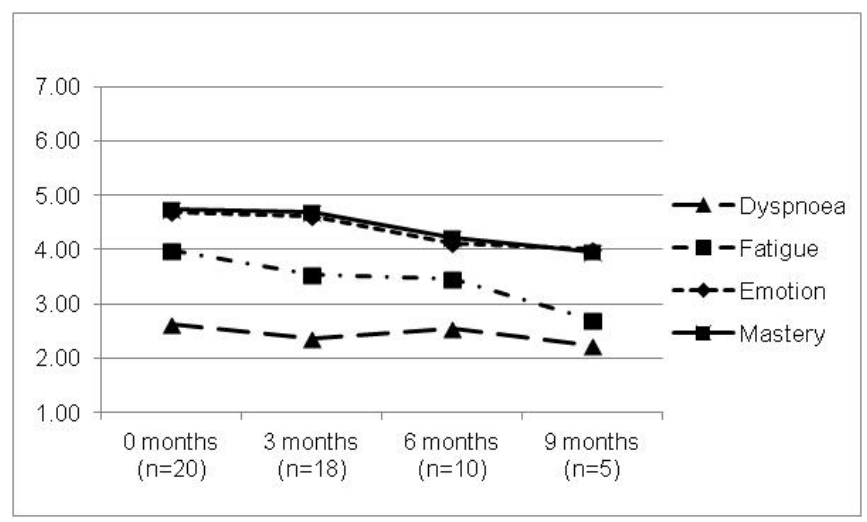

Abstract P135 Figure 1 Mean SR-CR0 scores in patients with at least 3 months follow-up 